\title{
Economic efficiency evaluation of merges and acquisitions in the sector of industry based on nonlinear model of synergistical growth of an industrial corporation value
}

\author{
A.E. Ivanov ${ }^{1, *}$, and $A . A$. Vasilchenko ${ }^{1}$ \\ ${ }^{1}$ South Ural State University, Chelyabinsk, Russia
}

\begin{abstract}
Numerous research works of the end of the 20th and of the beginning of the 21 st century prove that the synergistic effect often declared as the main goal of merges and acquisitions is not generated in fact. This is due to imperfection of the available methodology of its economic evaluation that does not take into account a nonlinear nature of the pooled corporation development. The article suggests a methodology of economic efficiency evaluation of merges and acquisitions in the sector of industry based on identification of synergistically successful acquisition order parameters. These are synergistic effects that with minimal investments in their achievement lead to a disproportionate increase in the value of an industrial corporation. A mathematical model has been created simulating the influence of these investments on the value of an industrial corporation. The model allows one to increase the degree of the decisions validity in merges and acquisitions in the sector of industry.
\end{abstract}

\section{Introduction}

In $1980 \mathrm{~s}-1990 \mathrm{~s}$ on the tide of rapid globalization processes, economists increasingly focused their attention on the economical efficiency of mergers and acquisitions (M\&A). Numerous research works of that period reveal that a significant part of mergers and acquisitions do not give the expected results. M. Bradley, A. Desai and E.H. Kim have found that only $7 \%$ of mergers and acquisitions lead to increment in value of the pooled companies [1]. J.W. Hunt in his study draws a conclusion that about $50 \%$ of all M\&A do not reach synergies [2]. M.L. Sirower shows that $70 \%$ of the acquisitions yield profit that is less than the premium paid for an acquired firm [3]. According to A. Agarwal and J. Jaffe's research, during the first years after M\&A the revenue of pooled companies reduces or remains unchanged [4].

An acquiring company often pays the price for a target firm which exceeds its net asset value. This overpaid amount is considered in the acquirer's financial statements as goodwill. It reflects the acquirer's expectations concerning the potential synergistic effects which may be generated as a result of merger or acquisition. At the beginning of XXI century a mandatory goodwill impairment testing was introduced in many International Financial Reporting Standards as well as many National Financial Reporting Standards. This made it possible to study the validity of M\&A initiators' expectations on the basis of information on the amounts recognized as a goodwill impairment. Recent studies show that a significant part of the purchased goodwill is impaired relatively soon $[5,6,7,8,9]$.

These results point out that the expectations of M\&A initiators with respect to the expected synergistic effect of integration are overestimated. This has to do with imperfection of the economical efficiency evaluation methodology of goodwill. Most of the synergistic effect valuating methods applied in planning M\&A are based on income approach to business valuation. Value of an enterprise according to income approach is considered as the present value of its future profits. The most common in business practice is the Excess Earnings Method [10]. The methods that are based on Edwards-Bell-Ohlson model and are supposed to use the combination of income approach and cost approach methodologies for business valuation are also often used [11]. A common disadvantage of all these methods is the linear logic that is the basis of future earnings forecast which expects a stable capitalization growth of pooled companies. However, an enterprise is an open system that interacts with the environment. The processes occurring in open systems are characterized by nonlinearity, no equilibrium and equifinality [12]. That is why the nonlinear nature of pooled companies development progress must be taken into account while forecasting future earnings growth rate.

\section{Methodology and results}

To determine the synergistic effect of an integration it is necessary to evaluate existing values of enterprises 
engaged in M\&A, and compare this amount with the present value of the industrial corporation formed as a result of the merger or the acquisition (we are going to use further the term "acquisition», considering merger as its particular case). We consider an industrial corporation as a sustainable combination of industrial and infrastructural objects that have a single owner and a common management body. Synergistic effect generating in open systems is caused by their emergence, i.e. appearance of some properties that are absent in isolated elements of the system. Synergistic effect for an industrial corporation results in its revenues increase or in its expenses reduction compared to the revenues and expenses of the acquiring company and the target company operating independently. An acquisition can be regarded as synergistically successful when integration of the acquiring and target companies generates a synergistic effect. We consider by synergistic effect the excess of the industrial corporation value over the sum total of values of isolated companies included in it. This article looks at acquisition implementation feasibility only from the point of view of synergistic effect generating. An acquisition can be economically effective, but fails synergistically if an industrial corporation value is equal to the sum total of values of isolated companies included in it. We have developed a methodology for economic evaluation of acquisitions feasibility in the sector of industry, which includes five stages.

1. Market value estimation of all acquisition participants as independently operating companies.

Discounted cash flow model is used for independently operating companies evaluation [13]. Equation (1) defines the present value of a company (PC) within this model.

$$
P C=\sum_{t=1}^{T} \frac{C F}{(1+r)^{t-1}}+\frac{T V}{(1+r)^{T}},
$$

where $C F$ - nominal incomes sum of a company; $t$ - a period of calculation (a year); $T$ - the forecast horizon; $r$ - the interest rate within the forecast period; $T V$ - the terminal value of a company in the post-forecast period.

2. Identification of potential synergy sources and determination of synergistically successful acquisition order parameters.

Open systems, which are far from equilibrium, depend on many external and internal environment factors and have unstable behaviour Their behaviour is determined by order parameters - leading variables that affect systems dynamics. Order parameters stimulate self-organization of a system, while the system's behaviour can vary greatly with insignificant alternation in the amount of the order parameters. Changes of other factors will have a much lesser impact on the behavior of a system, so their influence is negligible. We consider that the expected synergistic effect, which marks off the order parameters of synergistically successful acquisition are those that with minimal investment in their generating lead to a disproportionate increase in the value of an industrial corporation. For example, an acquisition is considered to generate two types of synergistic effects. The first one is the reduction of labor expenses by eliminating duplicate functions of administrative managers. The second is the sales revenues increase through implementation of highperformance incentive structure for sales managers, which is used at the target firm. Investing of $\$ 100$ to achieve the synergistic effect in the first case leads to $\$ 1,000$ expenses reduction. And a \$ 100 investment to generate the synergistic effect in the second case leads to $\$ 110$ revenues increase. In this example, it is the first synergistic effect that is the order parameter of synergistically successful acquisition.

In order to identify potential synergy sources and to determine order parameters of synergistically successful acquisition, a set of measures developed by A.E. Ivanov is suggested for use [14]. This toolkit is based on T.L. Saaty's analytic hierarchy process [15] and involves the use of expert assessment. Top managers of the company, who are planning an acquisition, may act out as experts for this purpose together with consultants, who are called for due diligence. The process of decision justification for an acquisition is represented as a hierarchical model. The goal of an industrial corporation in this model is the sustainable development, which can be reached by means of alternative development options. The acquisition of a target firm is one of these options. The criteria for goals achievement evaluation are the types of synergistic effects that can be generated in operating, financial and investing activities of an industrial corporation. At lower levels of this hierarchical model, experts make paired comparisons of the influence of merged companies' external and internal environment factors, on generating synergistic effects by Saaty's scale of relative importance. Thus, the experts can identify priority factors. At high levels of the hierarchical model, order parameters of synergistically successful acquisition are identified by evaluating their priorities vectors. Levelling of opinions in experts' groups is reached by calculating a geometric mean of priorities values. At the same time, an expert competence weighing system can be introduced as well as adjustments for relative compatibility of expert's estimation matrix.

3. Assessment of an industrial corporation value taking the synergistic effect into account.

Open systems develop nonlinearly. There are bifurcation points, where the performance of systems is unstable. An insignificant effect on the order parameter at bifurcation point can lead to the choice of further development of the system and to the phase transition. The logistic equation is one of the beststudied maps of the open systems development dynamics (2).

$$
X_{n+1}=C^{*} X_{n}\left(1-X_{n}\right)
$$

Logistic equation has an important property. If values of the constant $C$ are small, the argument $X_{n+1}$ 
increases exponentially. Large values of the constant $\mathrm{C}$ cause the cascade of bifurcations and the system drops down into the state of dynamic chaos. Thus, the system exponentially develops before the point of bifurcation and the dynamics of its development can be described by the Malthusian equation (3).

$$
\frac{d x}{d t}=\alpha x
$$

where $\frac{d x}{d t}$ - the change of argument $\mathrm{x}$ within a time period $\mathrm{t} ; \alpha$ - the growth coefficient.

Function (4) is the solution of the Malthusian equation.

$$
x=C e^{\alpha t},
$$

where $C$ - the constant.

An industrial corporation is an open system, so the dynamics of its value changes in the course of time can be described by means of logistic equation. At the same time, we believe that the forecast horizon for industrial corporation evaluation should be limited by the period between bifurcations. We've introduced this restriction, because it is difficult to reliably estimate the changes in the value of corporation at bifurcation points (e.g., during another acquisition process). Therefore, it leads to an unacceptably high risk taking into investor account evaluation while planning an acquisition [16]. Nonlinear equations are very sensitive to any initial conditions changing, so a qualitative forecast is only possible for a short period. A number of empirical studies conducted by McKinsey consulting group proves that synergistic effects are generated during several initial years after a merger or an acquisition $[17,18]$, so that the main increase in industrial corporation value by synergistic gains falls on this period. Thus, the industrial corporation value within the forecast horizon in the case of achieving synergies will be exponentially growing. Inserting the cash flows sum of separately operating companies in the solution of the Malthusian equation as the constant $\mathrm{C}$, we'll obtain the expression (5) to determine the value of an industrial corporation.

$$
\left.S P C_{T}=\sum_{i} P C_{i} * e^{\alpha}=\sum_{i}\left(\sum_{t=1}^{T} \frac{C F}{(1+r)^{t-1}}\right) * e^{\alpha}\right),
$$

where $S P C_{T}$ - the value of an industrial corporation, taking the synergistic effect into account and considering the cash flows within forecasting horizon; $P C_{i}$ - the cash flows sum total of an independently operating companies; $i$ - the number of pooling companies; $e^{\alpha}$ - the nonlinear function, which shows the ability of an industrial corporation to generate synergistic effect; $\alpha$ - the synergistic growth coefficient, which represents sensitivity of an industrial corporation value to investment in generating synergistic effect.

In this case the Malthusian equation describes only the dynamics of the industrial corporation changes in value within a framework of the forecast horizon. That is why the cash flow size of an industrial corporation within post-forecast horizon for the purpose of estimating the present value of an industrial corporation (SPC) is a separate item (6).

$$
\left.S P C=\sum_{i}\left(\sum_{t=1}^{T} \frac{C F}{(1+r)^{t-1}}\right) * e^{\alpha}+\frac{T V}{(1+r)^{T}}\right)
$$

Synergistic growth coefficient is determined from (7).

$$
\alpha=\left(\frac{d S E}{I d t}-1\right) *\left(\frac{d C F}{S E d t}-1\right) * \lambda,
$$

where $S E$ - the revenues increase (expenses reduction) of an industrial corporation as a result of investing in synergistic effect generating; $I$ - the volume of investment in synergistic effect generating; $\lambda-$ the synergistic effect attainability.

The first multiplier (7) shows synergistic effect sensitivity to investment into its achieving. The second multiplier (7) represents the sensitivity of an industrial corporation value to the amount of synergistic effect. To define multiplier we should compute the indicators for each of synergistic effects, which are the order parameters of integration. Then, the geometric mean of these results is calculated. The product of the first and the second multipliers (7) is the growth rate of an industrial corporation value under incrimination of investment in synergistic effects generating.

Possibility of adjusting achievement in synergistic effect is made by means of the third multiplier (7). We use the fuzzy sets theory described in L. Zadeh's works to estimate the possibility of synergistic effects generating. In recent years, methods based on fuzzy sets theory are often used for assessment of investment projects efficiency. In comparison with the classical probabilistic approaches, these methods allow one to take greater account of all the possible diversity of uncertainty types in forecasting development of large systems.

In fuzzy sets theory the possibility distribution is used instead of the probability distribution. The possibility distribution is described by compatibility function of a fuzzy number. The fuzzy number membership function is defined by means of linguistic variable whose values are not numbers but words (8).

$$
x=\langle x, T, U, G, M\rangle,
$$

where $x$ - the name of the variable; $T$ - the term-set of variable value, i.e. its linguistic value part; $U$ - the universe of discourse; $G$ - syntactic rule which defines the terms of the set T; $M-$ semantic compatibility rule when each linguistic value $\mathrm{x}$ is assigned its meaning according to this rule.

We have developed a linguistic variable "Attainability of integration synergistic effect". Its structure is shown in the Table 1 . 
Table 1. Structure of the linguistic variable "Attainability of integration synergistic effect".

\begin{tabular}{|c|c|c|}
\hline Element & Name & Content \\
\hline Variable name & $\mathrm{x}$ & Attainability of integration synergistic effect \\
\hline Syntactic rule & $\mathrm{G}$ & Very, higher, lower \\
\hline Set terms & $\mathrm{T}$ & High, medium, low \\
\hline $\begin{array}{c}\text { Semantic rule (the triangular form of a } \\
\text { fuzzy number is used for describing } \\
\text { compatibility function) }\end{array}$ & $\mathrm{M}$ & $\left(1-\frac{b-x}{b-a}, a \leq x \leq b ;\right.$ \\
$1-\frac{x-c}{c-b}, b \leq x \leq c ;$ \\
$0, x \prec a ;$ \\
$0, x \succ c$.
\end{tabular}

It makes sense to involve the experts, who previously helped to determine the order parameters, for estimating of synergistic effect attainability that are the order parameters of synergistically successful acquisition. The experts evaluate attainability of every single synergistic effect according to syntactic rule, e.g. "Attainability of labor expenses reduction by eliminating duplicate functions of managers is very high". These evaluations are shown as the fuzzy number $a_{i}$. As a result, we have $n$ fuzzy sets evaluations for $\mathrm{n}$ order parameters. The total evaluation of the most expected synergistic effect attainability interval is the convex combination of the fuzzy sets $a_{1}, \ldots, a_{n}$, i.e. the fuzzy set $A_{i}$ with the compatibility function (9).

$$
M_{A_{i}}(u)=M_{a_{1}}(u)+\ldots+M_{a_{n}}(u)
$$

The average opinion of the expert group is obtained by simple arithmetic averaging of compatibility functions values. The obtained limit defines the factor $\lambda$ (7).

The synergistic growth coefficient $\alpha$ (7) determines the growth rate of an industrial corporation value as a result of an acquisition.

If $\alpha<0$ an investment in synergistic effect generating leads to the growth rate decrease of the industrial corporation value, i.e. the acquisition is not feasible in terms of synergistic effect generating.

If $\alpha=0$ there is no synergistic effect because of one of the three possible reasons:

1) the growth rate of investments in generating synergistic effect and the revenues growth rate (expenses reduction rate) resulting from these investments are equal, that is typical for a linear developing system.

2 ) the growth rate of the industrial corporation value and the revenue growth rate (expenses reduction rate) caused by these investments in generating synergistic effect are equal, which is also typical for a linear developing system.

3) synergistic effect is unattainable either because it's impossible to generate it or because the order parameters of synergistically successful acquisition have been incorrectly defined.
If $\alpha>0$ an investment in synergistic effect generating leads to the growth rate increase of industrial corporation value, i.e. the acquisition is advisable in terms of synergistic effect generating. Thus, the values range of the corporation can be achieved with a high degree of confidence which is defined due to factor $\lambda$ (7) correction.

4. Attainable synergistic effect calculation

Attainable synergistic effect (SE) is determined from the formula (10).

$$
S E=S P C-\sum_{i} P C_{i}
$$

As the value of an industrial corporation is determined by range of values, so the attainable synergistic effect is determined by the range of values as well.

\section{Acquisition expenses evaluation}

If synergistic effect is attainable, it does not mean that the acquisition is advisable. One may say that it is advisable only if this synergistic effect exceeds acquisition expenses. Inequality (11) is the criterion of advisability of synergistically successful acquisition.

$$
S E>A E,
$$

where $A E$ - the acquisition expenses.

Acquisition expenses include:

- transaction costs, i.e. bargaining for acquisition costs, target firm evaluation costs, costs of financial audit of • the target firm, consultants fees etc.;

- severance payments for the target firm employees who have been laid off;

- expenses for the target firm managerial system restructuring;

- investments in synergistic effect generating.

These expenses can be extended in time, that's why we suggest evaluating them by means of discounted cash flow model. Thus, the difference between attainable synergistic effect and integration expenses will determine the values range of maximum allowable goodwill in the price structure of the target company. It makes no economic sense to pay to a target firm the amount that exceeds upper interval limit. 


\section{Practical relevance of research and conclusion}

Our methodology of economic efficiency evaluation of acquisitions in the sector of industry allows to enhance the validity of decisions in such transactions. Using our methodology, acquiring companies' decision makers will be able to compare attainable synergistic effect with the expenses of an acquisition. Limiting the number of synergistically successful acquisition order parameters provides an opportunity to target resources on achieving the most important synergistic effects. We realize that our methodology is limited by the period between bifurcations followed by limited forecast horizon. That is why studying the behavior of an industrial corporation value close to bifurcation points is an important area of our future researches.

The work was supported by Act 211 Government of the Russian Federation, contract № 02.A03.21.0011.

\section{References}

1. M. Bradley, A. Desai, E. Han Kim, Journal of financial economics, 21, 1, 3-40 (1988)

2. J.W. Hunt, Strategic management journal, 11, 1, 69-77 (1990)

3. M.L. Sirower, The synergy trap: how companies lose the acquisition game (The Free Press, USA, 1997)

4. A. Agrawal, J. Jaffe, Advances in mergers and acquisitions, 1, 7-41 (2000)

5. A.E. Ivanov, Economic analysis: theory and practice, 41, 35-59 (2015)
6. F. Gu, B. Lev, The accounting review, 86, 6, 1995-2022 (2011)

7. R. Camodeca, A. Almici, M. Bernardi, Problems and perspectives in management, 11, 3, 17-23 (2013)

8. W. Chen, P.K. Shroff, I. Zhang, Fair value accounting: consequences of booking marketdriven goodwill impairment (Working paper, SSRN, 2014)

9. Ch.E. Jordan, S.J. Clark, International journal of economics and finance, 7, 9, 159-168 (2015)

10. R.J. Alerding, The Excess Earnings Method, A BVR Webinar Handbook (2013)

11. J.A. Ohlson, Contemporary accounting research, 11, 2, 661-687 (1995)

12. H. Haken, Synergetics: Introduction and Advanced Topics (Springer, Germany, 2011)

13. A. Damodaran, Investment Valuation (John Wiley \& Sons, Inc., USA, 1996)

14. A.E. Ivanov, Economic analysis: theory and practice, 41, 60-70 (2013)

15. T.L. Saaty, The Analytic Hierarchy Process (McGraw-Hill, USA, 1980)

16. A.E. Ivanov, 42, 33-42 (2012)

17. M.M. Bekier, A.J. Bogardus, T. Oldham, The McKinsey quarterly, 4, 6-9 (2001)

18. S.A. Christofferson, R.S. McNish, D.L. Sias, McKinsey quarterly, 2, 92-99 (2004)

19. L.A. Zadeh, Information and control, 8, 3, 338353 (1965)

20. L.A. Zadeh, Information sciences, 1, 119-249 (1975) 